\title{
High frequency of Klinefelter syndrome in a cohort of Sri Lankan males with azoospermia and oligozoospermia
}

\author{
N. D. Sirisena ${ }^{1,2}$, U. M. J. E. Samaranayake ${ }^{1}$, A. P. Malalasekera ${ }^{1}$, V. H. W. Dissanayake ${ }^{1,2,3}$ \\ ${ }^{1}$ Department of Anatomy, Faculty of Medicine, University of Colombo, Sri Lanka \\ ${ }^{2}$ Human Genetics Unit, Faculty of Medicine, University of Colombo, Sri Lanka \\ ${ }^{3}$ Asiri Centre for Genomic and Regenerative Medicine, Asiri Surgical Hospital, Colombo, Sri Lanka
}

Keywords: Azoospermia; Karyotyping; Klinefelter syndrome; Male infertility; Oligozoospermia

\begin{abstract}
\section{Introduction}

Introduction: Klinefelter syndrome (KS) is the most common sex chromosomal aneuploidy. It affects 1:667 males. It is a major cause of male factor infertility due to the associated testicular atrophy. This study aims to describe the karyotype pattern identified in a cohort of Sri Lankan infertile males referred for cytogenetic testing as a component of the urological evaluation.
\end{abstract}

\section{Materials and methods}

The karyotype reports of males with severe oligozoospermia (sperm cell count $<5$ million cells/ml in seminal fluid analysis) and azoospermia, who were referred for karyotyping between January 2010 and February 2019 were maintained prospectively in an anonymized database and analysed retrospectively. All patients were referred from a single urological practice. Karyotyping was performed on routinely cultured lymphocytes after GTG-banding according to the guidelines of the International System for Human Cytogenetic Nomenclature (2016).

\section{Results}

A total of 69 infertile males underwent karyotyping. 40 (58.0\%) had azoospermia and 29 (42.0\%) had severe oligozoospermia. Abnormal karyotypes were seen in 14 (20.3\%) males, comprising of 11 (78.6\%) with KS (47, XXY) and $3(21.4 \%)$ with 46,XX karyotype. Polymerase chain reaction for $S R Y$ gene was positive in one male with 46,XX karyotype. Among the KS patients, 6 (54.6\%) had azoospermia and $5(45.5 \%)$ had severe oligozoospermia.

\footnotetext{
Correspondence: Nirmala D. Sirisena

E-mail:nirmala@anat.cmb.ac.lk

Received: 18-11-2019 Accepted: 24-12-2019

(iD) https://orcid.org/0000-0003-0994-6954 DOI: http://doi.org/10.4038/sljs.v37i4.8641
}

\section{Conclusions}

KS accounted for more than two-thirds of the chromosomal anomalies among the infertile males referred for karyotyping. These findings highlight the need for cytogenetic evaluation of severe oligozoospermic and azoospermic males prior to undertaking extensive invasive investigations and treatment measures in both partners.

\section{Introduction}

Klinefelter syndrome (KS) is the commonest sex chromosomal aneuploidy. It affects 1:667 males (1). Majority have the 47, XXY karyotype, while other mosaic and nonmosaic forms such as 48, XXXY, 49, XXXXY, $46, X Y / 47, X X Y$ have also been reported (2). The additional $X$ chromosomes cause abnormality in testicular germinal layer functions, leading to hypergonadotropic hypogonadism and male infertility (2).

Seminal fluid analysis (SFA) has a sensitivity rate of $89.6 \%$ in diagnosing male infertility and can identify 9 out of 10 infertile men (3). KS individuals are known to have severe oligozoospermia or azoospermia in their SFA (4). Thus, karyotyping is routinely carried out in developed countries when either severe oligozoospermia or azoospermia are observed in the SFA, with a view to detecting underlying genetic conditions such as $\mathrm{KS}(5,6)$.

Yoshida et al., reported significantly higher chromosomal abnormalities in males with low testicular volume, and high levels of serum follicular stimulating hormone (FSH) or luteinizing hormone ( $\mathrm{LH})(7)$. It is noteworthy that the clinical features of hypogonadism and other laboratory findings such as raised serum FSH and LH levels are taken into consideration when deciding on recommendations for cytogenetic testing.

At present, there is paucity of data on the frequency of Klinefelter syndrome among Sri Lankan infertile males. Currently, there are also no national guidelines for cytogenetic testing of such individuals in our health care setting. This study, therefore aims to describe the karyotype pattern identified in a cohort of Sri Lankan infertile males referred for cytogenetic testing as a component of the urological evaluation. 


\section{Materials and methods}

The karyotype reports of Sri Lankan males diagnosed with severe oligozoospermia (sperm count $<5$ million cells $/ \mathrm{ml}$ ) or azoospermia, who were referred for cytogenetic testing between January 2010 and February 2019 were maintained prospectively in an anonymized database and was analysed retrospectively. All patients were referred from a single urological practice. The study was approved by the Ethics Review Committee of the Faculty of Medicine, University of Colombo (Protocol no. EC-13-181).

Conventional karyotyping was performed by an experienced cytogeneticist on peripheral blood lymphocytes after routine chromosome culture and GTG-banding according to the guidelines of the International System for Human Cytogenetic Nomenclature (2016). In each case, a minimum of 20 well-banded metaphase spreads were examined at 450-500 resolution to identify the presence of any numerical or structural abnormalities. In individuals with the 46,XX karyotype, polymerase chain reaction (PCR) for the presence of the sex-determining region on the $\mathrm{Y}$ chromosome (SRY) gene was performed using standard published protocols by Ke-hui-cui (8).

\section{Results}

Out of the total 69 infertile males who underwent karyotyping, 40 (58.0\%) had azoospermia and 29 (42.0\%) had severe oligozoospermia. Abnormal karyotypes were seen in $14(20.3 \%)$ males, comprising of $11(78.6 \%)$ with KS (47, XXY) and 3 (21.4\%) with 46, XX karyotype. Among the KS patients, $6(54.6 \%)$ had azoospermia and $5(45.5 \%)$ had severe oligozoospermia. PCR for $S R Y$ gene was positive in one male with the 46, XX karyotype, due to $S R Y$ translocation to the X-chromosome, and the other two were negative for $S R Y$ gene. The karyotype data are shown in Table 1.

Table 1. Karyotypes identified in azoospermic and severe oligozoospermic males

\begin{tabular}{|l|l|l|}
\hline \multicolumn{3}{|l|}{ Karyotypes identified in azoospermic males } \\
\hline Semen analysis & Karyotype & Number of patients (\%) \\
\hline \multirow{3}{*}{ Azoospermia } & $46, \mathrm{XY}$ & $31(77.5 \%)$ \\
\cline { 2 - 3 } & $47, \mathrm{XXY}$ & $6(15.0 \%)$ \\
\cline { 2 - 3 } & $46, \mathrm{XX}$ & $3(7.5 \%)$ \\
\hline Total & \multicolumn{2}{|l|}{40} \\
\hline Karyotypes identified in severe oligozoospermic males \\
\hline Severe & $46, \mathrm{XY}$ & $24(82.8 \%)$ \\
\hline Oligozoospermia & $47, \mathrm{XXY}$ & $5(17.2 \%)$ \\
\hline Total & & 29 \\
\hline
\end{tabular}

\section{Discussion}

Among the numerous factors causing male infertility, underlying chromosomal abnormalities are known to play a prime role. The clinical phenotypes associated with these chromosomal anomalies may not be apparent during the early years of life leading to presentation of these males later in their adulthood with infertility.

Wosnitzer reported that $3 \%-5 \%$ of males with severe oligozoospermia and $14 \%-19 \%$ of males with azoospermia in their SFA had abnormal karyotypes, with the majority being non-mosaic KS (9). Similarly, a Japanese study conducted by Nakamura et al., identified that $12.6 \%$ (225/1790) infertile males had chromosomal abnormalities, and the most frequent anomaly was KS in $28.4 \%$ (64/225) (10). In the present study, one fifths of the cohort had abnormal karyotypes and KS accounted for more than two-thirds of the chromosomal anomalies among the infertile males. However, a study limitation that may have contributed to the high frequency of $\mathrm{KS}$ observed in this cohort might be that it was performed on infertile males referred from only a single urological practice.

KS (47,XXY) characteristically presents with hypogonadism, raised FSH, LH and low testosterone levels (9). Azoospermia was observed in $54.6 \%$ and severe oligozoospermia in $45.5 \%$ of males with KS in the present study. Several other studies have also reported KS to be associated predominantly with azoospermia and severe oligozoospermia (11). European and American guidelines recommend performing karyotyping and genetic counseling in males who present with severe oligozoospermia and azoospermia $(5,6)$. The European Association of Urology (EAU) guidelines 2016, recommends performing standard karyotype analysis in all men with sperm count $<10$ million $/ \mathrm{ml}$ who seek fertility treatment (5). The American Urological Association (AUA) recommends carrying out karyotyping in patients with much lower sperm counts $(<5$ million/ml). Infertile males who have had a past history of fertility and those who have had prior sperm counts above 5 million cells $/ \mathrm{ml}$ in SFA are usually not recommended to undergo cytogenetic testing (6). Prognostic information for guiding fertility treatment is a necessity in $\mathrm{KS}$ patients as the available treatment modalities are not only expensive but also undergoing such interventions are emotionally and psychologically taxing for the affected couples.

Apart from KS, individuals who are phenotypically male with the 46,XX karyotype may also present with infertility. One such patient in the present study had 46, XX karyotype and the PCR for the SRY gene was positive. This finding along with the patient's clinical phenotype, which included small testicular volume and azoospermia were consistent with De La Chapelle syndrome. Two other males with the 46,XX karyotype tested negative for the SRY gene using PCR. 
In such cases, SRY gene sequencing would need to be performed to identify point mutations which are not detectable by PCR. Majority of the 46,XX males are known to be azoospermic. A similar finding was observed in our study.

The paucity of data on the chromosomal abnormalities underlying male factor infertility and lack of established national guidelines on cytogenetic evaluation of such males are current challenges facing the practice of fertility treatment in Sri Lanka. The high prevalence of KS in this cohort highlights the need for cytogenetic testing of severe oligozoospermic and azoospermic males to help avoid extensive and invasive investigations in both partners and unnecessary physical and psychological burden on them.

All authors disclose no conflict of interest. The study was conducted in accordance with the ethical standards of the relevant institutional or national ethics committee and the Helsinki Declaration of 1975, as revised in 2000

\section{References}

1. Bojesen A, Juul S and Gravholt CH. Prenatal and postnatal prevalence of Klinefelter syndrome: a national registry study. The Journal of Clinical Endocrinology\&Metabolism. 2003;88:622-6. https://doi.org/10.1210/jc.2002-021491

2. Los E FG. Klinefelter Syndrome. Treasure Island (FL): StatPearls Publishing; Jan 2019.

3. Butt F and Akram N. Semen analysis parameters: Experiences \& insight into male infertility at a tertiary care hospital in Punjab. J Pak Med Assoc. 2013;63:558-62.
4. Foresta C, Garolla A, Bartoloni L, Bettella A and Ferlin A. Genetic abnormalities among severely oligospermic men who are candidates for intracytoplasmic sperm injection. The Journal of Clinical Endocrinology \& Metabolism. 2005;90:152-6. https://doi.org/10.1210/jc.2004-1469

5. Dohle G, Colpi G, Hargreave T, Papp G, Jungwirth A, Weidner W, et al. EAU guidelines on male infertility. European Urology. 2005;48:703-11. https://doi.org/10.1016/j.eururo.2005.06.002

6. American Urological Association Education and Research I. The optimal evaluation of the infertile male: AUA best practice statement 2010.

7. Yoshida A, Miura K and Shirai M. Cytogenetic survey of 1,007 infertile males. Urologia Internationalis. 1997;58:166-76. https://doi.org/10.1159/000282975

8. Cui KH. Size differences between human X and Y spermatozoa and prefertilization diagnosis. Molecular Human Reproduction. 1997;3:61-7. https://doi.org/10.1093/molehr/3.1.61

9. Wosnitzer MS. Genetic evaluation of male infertility. Translational Andrology and Urology. 2014;3:17. doi: 10.3978/j.issn.2223-4683.2014.02.04

10.Nakamura Y, Kitamura M, Nishimura K, Koga M, Kondoh N, Takeyama M, et al. Chromosomal variants among 1790 infertile men. International Journal of Urology. 2001; 8:49-52. https://doi.org/10.1046/j.1442-2042.2001.00242.x

11.Arafa MM, Majzoub A, AlSaid SS, El Ansari W, Al Ansari A, Elbardisi Y, et al. Chromosomal abnormalities in infertile men with azoospermia and severe oligozoospermia in Qatar and their association with sperm retrieval intracytoplasmic sperm injection outcomes. Arab Journal of Urology. 2018;16:132-9. https://dx.doi.org/10.1016\%2Fj.aju.2017.11.009 